\title{
Gráfico de função polinomial: uma discussão sobre dificuldades de aprendizagem no Ensino Superior
}

\section{Polynomial functions graphs: discussing college students' difficulties learning}

\author{
Cinthya Maria Schneider Meneghetti, Bárbara Denicol do Amaral Rodriguez e \\ Cristiana Andrade Pofffal \\ cinthya.schneider@gmail.com; barbararodriguez@furg.br; cristianaandrade@furg.br
}

Universidade Federal do Rio Grande - FURG, Brasil

\section{Resumo}

Este artigo apresenta e discute dificuldades de alunos do primeiro ano de cursos de Ciências Exatas e Engenharias da Universidade Federal do Rio Grande (FURG) no que diz respeito à leitura e compreensão de um gráfico de uma função polinomial. A partir de um estudo qualitativo dos dados obtidos e das atividades propostas, pretende-se identificar algumas lacunas na aprendizagem e analisar a falta de domínio dos conteúdos envolvidos. Muitas vezes, os alunos não estão aptos e não avançam nas disciplinas da graduação, pois não possuem os pré-requisitos necessários para dar continuidade ao processo de ensino-aprendizagem. Através de um teste de sondagem, foi possível investigar alguns conhecimentos matemáticos prévios e as principais dúvidas dos alunos. Os resultados deste trabalho permitem traçar uma estratégia inicial para dar continuidade e complementar a formação básica do aluno. Portanto, propõem-se atividades utilizando o software GeoGebra para trabalhar os conceitos envolvidos e auxiliar na transição do Ensino Médio para o Ensino Superior. Ao final, ainda discute-se a aplicação de uma das atividades propostas em uma turma.

Palavras-chave: Representação gráfica, função polinomial, erros frequentes.

\section{Abstract}

This paper presents and discusses Engineering and Sciences freshmen difficults related to polynomial graph reading and comprenhension at FURG (Universidade Federal do Rio Grande). Analyzing qualitative data and the proposed activities, we intend to identify learning problems and the lack of mastery of the math concepts studied during elementary and high school. Frequently, students are not prepared and do not approve the graduation courses, as they have significant gaps in their academic fundamental training which is required to learning. By means of a test, we investigate students' math prior knowledge and their main doubts. The results allow preparing strategies in order to continue and complement their basic education. We also suggest computational activities envolving polynomial functions using Geogebra in the interest of assisting the trasition from High School to the University. Finally, we discuss the application of one of the propposed activities to a group of students.

Keywords: graph, polynomial function, common mistakes. 


\section{Introdução}

As disciplinas de matemática no Ensino Superior objetivam fornecer aos estudantes as ferramentas necessárias para a modelagem e resolução de problemas. A partir disso, o profissional deverá ser capaz de avaliar e prever novas situações em seu campo de trabalho. Segundo Pereira et al. (2012), as disciplinas matemáticas auxiliam no desenvolvimento do pensamento lógico do futuro profissional. Ao concluir sua graduação e ingressar no mercado de trabalho, estará apto a utilizar o raciocínio e as ferramentas matemáticas para trabalhar com simulações, lidar com os dimensionamentos de seus projetos e buscar novas soluções para os problemas com os quais se defrontar. De acordo com Simmons (1987), todos os professores sabem que os alunos tremem diante de problemas contextualizados, pois usualmente exigem pensamento não rotineiro. Entretanto, a utilidade da matemática nas várias Ciências demanda uma iniciativa dos professores: ensinar aos estudantes como compreender o significado de um problema dentro de um contexto, julgar o que é relevante e traduzir as palavras para esboços e equações que auxiliarão na determinação da solução final.

O estudo das disciplinas de matemática nos cursos de Ciências Exatas e das Engenharias, normalmente, ocorre junto às disciplinas que compõem o chamado núcleo básico, sendo ministradas nos dois primeiros anos desses cursos. Nestas disciplinas observa-se uma alta taxa de insucesso e evasão. Estes fatos preocupantes têm levado pesquisadores e professores a debater a respeito do processo de ensino e aprendizagem dos conhecimentos matemáticos nos diversos níveis de ensino, entre eles, o Ensino Superior (Malta (2004)).

Objetivando investigar as possíveis causas para estas elevadas taxas, diversas pesquisas apontam algumas dificuldades apresentadas pelos alunos que ingressam a cada ano nos cursos de Ciências Exatas e Engenharias em Instituições de Ensino Superior. Entre elas, podemos destacar as dificuldades na resolução de exercícios envolvendo funções, principalmente no que diz respeito a sua interpretação dentro de um contexto e a sua representação gráfica. Dentre os vários conteúdos matemáticos abordados no Ensino Médio, o conceito de função é um dos primeiros a ser tratado e, sem dúvida, o mais importante. Segundo da Cruz Mendes et al. (2012), o emprego de funções quadráticas, por exemplo, a partir de problemas contextualizados, torna a aprendizagem matemática um meio de atender às necessidades da sociedade. O estudo de funções favorece a integração entre diferentes campos da Matemática e está presente nos mais diversos ramos das Ciências, sendo um conceito importante na modelagem de diversos problemas em diferentes áreas do conhecimento.

No Ensino Superior, o estudo de funções, particularmente as funções polinomiais, é um dos pré-requisitos para a disciplina de Cálculo Diferencial e Integral. Em seu livro, Edwards (2006) destaca que durante os últimos 300 anos, à medida que o Cálculo se desenvolvia até atingir a forma atual, muitas funções se revelaram de grande importância em modelos da vida real, dentre elas destacam-se as funções polinomiais. Hoffmann e Bradley (1999) complementam que a função polinomial está presente em todo o Cálculo, com exemplos e cumprindo um papel útil em diversas aplicações práticas.

O aumento do número de alunos que reprovam ou evadem nas disciplinas de Cálculo Diferencial e Integral da Universidade Federal do Rio Grande (FURG) tem motivado os docentes do Instituto de Matemática, Estatística e Física (IMEF) a investigar as causas deste fato (Rodriguez et al. (2015)). São diversas as razões para a alta evasão em cursos de Ciências Exatas e Engenharias no Ensino Superior. Segundo Catani et al. (2001), uma das causas da evasão dos cursos de graduação era devida à pouca flexibilidade dos currículos escolares diante das mudanças do mercado de trabalho e por esta razão tornavam os cursos desatualizados e desinteressantes para os estudantes. Pereira et al. (2012) sugere que muitos alunos que ingressam nesses cursos acabam desistindo dos mesmos devido a sua inabilidade em trabalhar questões matemáticas. Para Rezende (2003), grande parte das dificuldades é consequência da falta de preparação, na Educação Básica, em alguns conteúdos matemáticos, entre eles funções polinomiais. Tais dificuldades podem estar associadas a maneira como o conteúdo é abordado. Segundo Ballejo (2009), os exercícios apresentados, por muitos professores e livros didáticos, junto ao ensino de polinômios, por exemplo, têm caráter manipulativo e de memorização, na medida que exigem pouco raciocínio e muita aplicação direta de definições, teoremas e fórmulas. Por outro lado, segundo Fainguelernt e Nunes (2012), existe uma tendência de se exigir cada vez menos memorização de fórmulas e valorizar a autonomia dos alunos. Por exemplo, o Exame Nacional do Ensino Médio (ENEM) criado em 1998 pelo INEP/MEC para avaliar o desempenho dos estudantes brasileiros ao término da Educação Básica (Brasil (2016)), é uma prova contextualizada e interdisciplinar que exige que o estudante saiba interpretar, transferir e aplicar conteúdos de matemática estudados em diferentes situações-problema.

Já em 2004, Soares e Sauer (2004) alertavam para a necessidade de investigar problemas relacionados às disciplinas de matemática lecionadas nos anos iniciais dos cursos de graduação. A investigação do modelo de ensino adotado nos cursos de graduação se faz necessária uma vez que grande parte dos planos de ensino dessas disciplinas ainda não possuem caráter interdisciplinar e contextualizado. 
Segundo Pereira et al. (2012), entre as linhas de pesquisa para tentar entender e superar as dificuldades, estão o trabalho com o processo de ensino e aprendizagem que ocorre nas escolas, o estudo das teorias de aprendizagem, a investigação dos currículos dos cursos e das disciplinas, a pesquisa na aplicação da tecnologia de informação e comunicação no ensino, o emprego de metodologias diferenciadas, a tentativa de levar conceitos trabalhados nas faculdades para anos anteriores ao ingresso nas mesmas, o estudo do aspecto histórico do assunto considerado, a análise das formas de avaliação, dentre outros.

Buscando contemplar algumas destas linhas de pesquisa, neste trabalho são apresentados os dados obtidos a partir de uma sondagem realizada com alunos do primeiro ano de cursos de Ciências Exatas e Engenharias da FURG. O objetivo da sondagem é buscar entender os tipos de dúvidas que os alunos apresentam nas disciplinas de Cálculo Diferencial e Integral no que diz respeito à leitura e compreensão de um gráfico de uma função polinomial, a fim de traçar uma estratégia inicial para dar continuidade e complementar a formação básica do aluno.

Além disso, espera-se, a partir desta experiência, desenvolver práticas pedagógicas alternativas com a finalidade de auxiliar na diminuição da retenção e evasão nas disciplinas introdutórias de Matemática. Nesse primeiro momento da investigação as atenções estarão voltadas aos aspectos referentes ao conhecimento matemático e, portanto, sem um maior aprofundamento na influência de outros fatores no processo de aprendizagem.

Segundo Cury (2007), analisar as respostas produzidas pelos alunos em qualquer conteúdo, é uma das formas de auxiliá-los a construir o conhecimento básico necessário para transitar pelos conteúdos específicos de suas áreas de formação. A autora afirma ainda que é possível entender como ocorre o processo de construção do conhecimento por parte dos alunos através de suas produções escritas e, a partir delas, é possível compreender as dificuldades apresentadas por eles com relação aos conteúdos.

Com essa compreensão torna-se viável a elaboração de estratégias efetivas para a superação de tais dificuldades e, portanto, seguir as linhas propostas por Pereira et al. (2012) que tratam da aplicação da tecnologia de informação e comunicação no ensino e o emprego de metodologias diferenciadas. Dentro deste contexto, ao final do artigo, são propostas quatro atividades que buscam retomar e pontuar aspectos relativos aos conteúdos envolvidos no teste de sondagem aplicado. Tais atividades têm como objetivo inserir a tecnologia da informática como parte da metodologia de ensino e propor aos alunos situações nas quais eles possam aprender com suas descobertas, contribuindo, assim, para o desenvolvimento do raciocínio e da habilidade de abstração. Inicialmente é apresentada uma discussão sobre a bibliografia atual. A fim de investigar a metodologia proposta, são apresentados resultados de uma análise inicial obtidos a partir da aplicação da atividade 4 em um grupo de alunos de um curso de Ciências Exatas da FURG. Para finalizar discute-se a importância da aprendizagem de diferentes formas de representação de objetos matemáticos e propõe-se uma diferente abordagem pedagógica.

\section{Uma breve discussão sobre a bibliografia atual}

Eisenberg e Dreyfus (1994) e Coxford e Shulte (1994) consideram que os polinômios são importantes e devem fazer parte dos currículos escolares. Para Lima et al. (2001), muitos autores dão aos polinômios um tratamento exclusivamente algébrico, não apresentando qualquer gráfico. Ele ainda ressalta que os polinômios devem ser estudados sob o ponto de vista algébrico, geométrico e também numérico. A abordagem que prioriza somente os cálculos e não a representação gráfica desconsidera o fato de que ler e interpretar um gráfico é uma tarefa presente nas atividades diárias não só para um profissional de Ciências Exatas e Engenharias. Os gráficos ilustram fenômenos cotidianos, como a produção e distribuição de produtos, custos, lucro e vendas ou ainda, o cálculo da quantia que pagamos a cada mês na nossa conta de luz ou o preço que pagamos em uma corrida de táxi. Os alunos, de maneira geral, apresentam grande dificuldade em ler e interpretar o gráfico de uma função, não reconhecendo, por exemplo, o intervalo numérico no qual a função assume valores positivos ou negativos (Questão 1).

As dificuldades apresentadas na construção de gráficos de funções polinomiais, por parte do aluno, não estão relacionadas diretamente ao cálculo dos elementos necessários a tal construção, mas a compreensão da representação desses elementos no plano cartesiano e no comportamento de cada curva em seus diversos intervalos. No que diz respeito aos obstáculos para a aprendizagem de gráficos, concorda-se com Nasser et al. (2012), que diz que os estudantes acreditam que o gráfico de uma função é obtido marcando alguns pontos no plano cartesiano e unindo-os por segmentos de reta, deixando de considerar a lei de formação da função.

Dierings (2014) afirma que livros utilizados no Ensino Médio como Matemática, Dante (2010), Matemática completa, Giovanni et al. (2005), Matemática aula por aula, Filho e Silva (2000), e Matemática para o ensino médio, Fernandes et al. (2005) abordam os conteúdos: Definição de polinômios; Grau de polinômio; Valor numérico de polinômio; Identidade de polinômios; Operações com polinômios; Dispositivo prático de Briot-Ruffini; Teorema do 
Resto e Relações de Girard. Segundo Dierings (2014), talvez pela falta de incentivo do uso de tecnologia não se trabalhe com traçado de gráficos. O autor afirma ainda que o fato de usar o traçado de gráficos, mesmo com apoio de programas computacionais, seria uma ferramenta importante para o desenvolvimento da noção de localização de uma ou mais raízes reais de uma função polinomial.

De fato, é possível perceber através dos conteúdos listados que os livros didáticos citados contemplam e enfatizam a álgebra dos polinômios e funções polinomiais e pouco abordam o estudo e traçado de gráficos. Também nos livros de Cálculo adotados nas universidades brasileiras, observa-se que não há ênfase na intuição gráfica, usam as derivadas como ferramenta principal para a construção gráfica. O livro Cálculo, Thomas (2009), apresenta uma revisão acerca do traçado do gráfico de funções, mas sugere o uso da derivada para determinar o formato de uma curva entre os pontos do gráfico. Mostra os gráficos de $f(x)=x^{n}$, para $n=1,2,3,4,5$. Explica que à medida que a potência $n$ fica maior, as curvas tendem a se achatar sobre o eixo $x$ no intervalo $(-1,1)$ e também a subir repentinamente para $|x|>1$. Comenta que os gráficos de função polinomial $p(x)=a_{n} x^{n}+\ldots+a_{1} x+a_{0}$, onde $a_{n} \in \mathbb{R}$ são os coeficientes de $p(x)$, são ensinados após o aprendizados das derivadas.

O livro Cálculo: um novo horizonte, Anton (2000), define a função potência $f(x)=x^{p}$ e para $p \in \mathbb{Z}_{+}$, então a função potência tem a forma $y=x^{n}$ para $n \in[1,5]$, comenta sobre paridade, simetria e comportamento do gráfico, fornecendo uma ideia intuitiva dos limites analisando os intervalos $-1<x<1, x>1$ e $x<-1$.

O exemplar de Munem e Foulis (1982) apresenta um capítulo dedicado a uma revisão de funções, mas não há comentários sobre o esboço de funções polinomiais de grau $n$. O primeiro volume da coleção Um Curso de Cálculo de Guidorizzi (2006), no capítulo de funções apresenta o gráfico de $y=x^{3}$, comenta sobre o crescimento de $y$ à medida que $x$ cresce e à medida que $x$ se aproxima de zero. Utiliza a translação para traçar gráficos de $g(x)=(x-1)^{3}$ e $f(x)=x^{3}-1$. Simmons (1987) tem no primeiro capítulo uma revisão sobre números, funções e gráficos. Comenta que não é possível esboçar gráficos de funções polinomiais com a localização precisa dos pontos de máximos e mínimos. Utiliza o estudo do sinal da função através da forma fatorada e usa a noção intuitiva de limite ao se referir ao comportamento gráfico. Em Cálculo A, de Fleming (2006), o capítulo de funções sugere a construção de uma tabela para determinar o gráfico de uma função e comenta que técnicas serão abordadas com o estudo de derivadas. Não apresenta gráficos de funções polinomiais de grau maior que 2.

É importante ressaltar que os livros de cálculo analisados não abordam inicialmente o estudo de funções polinomiais da forma $f(x)=a_{n} x^{n}+\ldots+a_{1} x+a_{0}$ bem como o seu gráfico. O comportamento da função é estudado somente nos capítulos de aplicações de derivadas. No entanto, acredita-se que o aluno ao ingressar nos cursos de ciências exatas e engenharias da universidade poderia esboçar o gráfico, estudar o sinal da função e sua forma ao longo do domínio antes de estudar as técnicas de derivação e os testes da primeira e segunda derivada. Também, poderia, a partir do gráfico, extrair informações sobre a função (mesmo sem explicitar sua lei) e não apenas o contrário.

\section{Descrição da pesquisa: aplicação de teste de sondagem}

A pesquisa foi realizada nas dependências da Universidade Federal do Rio Grande (FURG) no mês de março de 2015, em sala de aula e na presença dos professores. Antes da entrega do teste de sondagem de conhecimentos, explicou-se a natureza da pesquisa e os alunos aderiram voluntariamente ao processo de investigação. Não foram produzidos vídeos, nem fotografias.

O teste foi elaborado de modo a conter perguntas simples e de fácil entendimento; relativamente curto, para não se tornar cansativo e fazer com que o aluno se sentisse desestimulado a responder até o fim. De um modo geral, as questões buscam caracterizar as particularidades da formação matemática dos alunos. Neste relato discutem-se as respostas dos alunos em questões que compreendem leitura e interpretação de gráficos e extrair dele as informações solicitadas. Além disso, são analisadas as soluções relacionadas as questões de funções polinomiais na sua forma geral e em uma aplicação. Normalmente, no Ensino Médio, são estudados gráficos de funções polinomiais de grau um e dois. No teste de sondagem aplicado, o gráfico apresentado é de uma função polinomial de grau três.

Com o gráfico escolhido pretende-se também investigar a capacidade dos alunos de generalizar conceitos sobre funções, ideias que percorrem o conhecimento escolar desde as primeiras noções de proporcionalidade nas séries iniciais do Ensino Fundamental, e que já foram estudados como: o sinal de uma função, as raízes e intersecção do gráfico com o eixo das ordenadas. Tais conceitos são amplamente discutidos na Educação Básica para funções polinomiais de graus um e dois e são pré-requisitos para o estudo dos conteúdos da disciplina de Cálculo Diferencial e Integral. A teoria matemática acerca das funções polinomiais de graus um e dois pode ser entendida como o saber prévio do aluno, a base para os novos saberes a serem trabalhados, neste caso, as funções polinomiais de grau três. 
Segundo Cury (2007), se o estudante conhece o comportamento do gráfico de uma função, ele pode antever o que acontece em determinados pontos, saber onde ela é contínua, onde é derivável, onde apresenta pontos de extremos locais ou absolutos, etc.

Baseado no resultado da análise dos principais erros será possível repensar e priorizar o estudo dos conceitos que ainda não foram completamente compreendidos pelos discentes e, assim, pensar alternativas para melhorar o aproveitamento dos alunos nas disciplinas de Cálculo. Segundo Cavasotto e Portanova (2008), a análise de erros enquanto linha de pesquisa pode ter um caráter diagnóstico, possibilitando a compreensão das dificuldades na aprendizagem apresentadas pelos estudantes.

A seguir, são apresentadas algumas das questões propostas, seguida da descrição das respostas obtidas e uma breve discussão dos resultados.

\section{Apresentação dos dados}

Nesta seção são enunciadas três questões propostas e faremos a análise das respostas dos discentes.

Questão 1. Observe o gráfico da Figura 1 e responda:

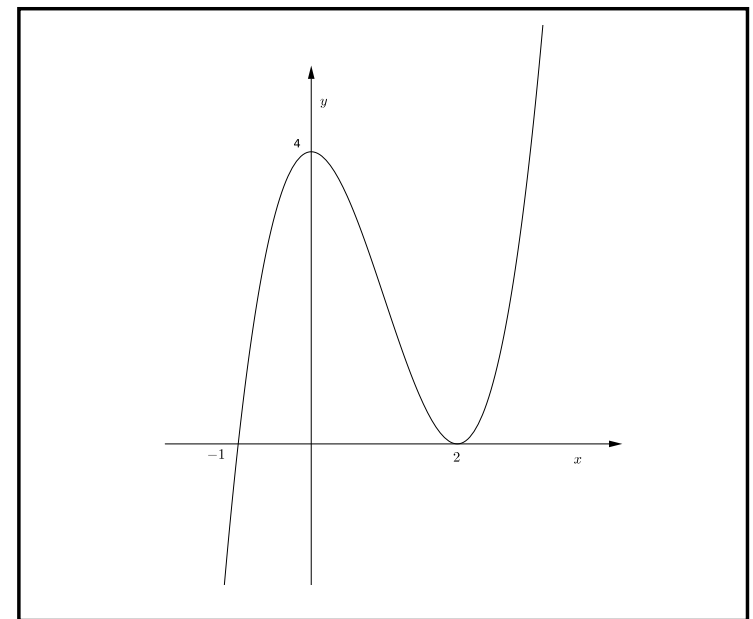

Figura 1: Gráfico da função polinomial Questão 1.

a) para quais valores de $x$ tem-se $f(x)>0$ ?

b) para quais valores de $x$ tem-se $f(x)=0$ ?

c) para quais valores de $x$ tem-se $f(x)<0$ ?

d) qual é o valor de $y$ quando $x=0$ ?

Responderam a Questão 1, 55 alunos matriculados no primeiro ano de cursos de graduação de Ciências Exatas e Engenharias da FURG. Acertaram integralmente a questão 10 alunos (aproximadamente 18\%). Não responderam a questão, 12 alunos.

Com relação ao item a) percebeu-se que alguns alunos não compreendem o significado das desigualdades. Precisamente, não conseguiram entender a diferença entre escrever $f(x)>0$ e $f(x)<0$. É possível perceber que estes alunos desconhecem a leitura correta da desigualdade. Entre as respostas, houve aqueles que escreveram que a solução seria a desigualdade $x>+\infty$ ou o intervalo ] $-1,+\infty$ ] apresentando, em ambos os casos, o infinito como um número real. Outro fato relevante é o de que 5 alunos responderam de maneira incorreta que a solução é $x>-1$. No entanto, essa resposta desconsidera o fato de que $f(2)=0$. Além disso, 7 alunos responderam $x=2$, evidenciando que não sabem interpretar a pergunta e desconhecem a solução da inequação em questão.

Com relação ao item b), 5 alunos responderam corretamente, mas utilizaram uma notação incorreta para exibi-la. As raízes são $x=-1$ e $x=2$. No entanto, foram apresentadas respostas como: $(-1,2),[1,2],[-1]$ e [2]. Note que a primeira delas pode representar um ponto no plano cartesiano ou ainda um intervalo aberto. A segunda, um 
intervalo fechado e as demais uma notação algébrica que representa a classe de equivalência do número e que naturalmente não se aplica à situação em questão. Observe que, propositalmente, não foi perguntado "para quais valores de $x$ tem-se $y=0$ ?", pois acredita-se que muitos alunos sabem resolver a equação, mas não associam a solução algébrica com sua interpretação geométrica.

Com relação ao item c), observa-se que 2 alunos inverteram o intervalo e a abertura dos colchetes, escrevendo $[-1,-\infty]$ em vez de $]-\infty,-1[$. Frequentemente nas aulas de Cálculo, os alunos manifestam sua dificuldade em representar intervalos utilizando parênteses e colchetes. A maioria prefere a notação por compreensão, isto é, com desigualdades (mesmo sem apresentar o domínio completo) ou ainda, a representação geométrica, que consiste em desenhar a reta numérica real e destacar o intervalo em questão. Alguns alunos, ainda, não compreendem a densidade do conjunto dos números reais e não reconhecem a ordenação na reta real, ou seja, fixado um ponto $O$ chamado de origem e correspondente ao número 0 (zero); os pontos situados a direita de $O$ constituem a semirreta positiva e os pontos situados a esquerda de $O$, formam a semirreta negativa (Lima (1998)).

$\mathrm{O}$ item $\mathrm{d}$ ) foi o que obteve maior número de acertos entre os alunos que responderam a questão. Somente 11 alunos erraram. Apenas para 2 alunos, a imagem de um ponto já parece ser conteúdo aprendido. Apesar de ter sido o item com o maior número de acertos, a localização de pontos no plano cartesiano em que somente a abscissa ou a ordenada vale zero é um assunto no qual os alunos também apresentam dúvidas.

Essencialmente a dificuldade presente na resolução desta questão refere-se a transposição da representação geométrica da função para a representação analítica das soluções das inequações e equações do problema.

Normalmente, no Ensino Médio, os professores propõem listas de exercícios em que o discente resolve mecanicamente inequações e equações sem associar a solução ao seu significado geométrico. No Ensino Superior, esta habilidade é requisitada nas disciplinas iniciais e, o aluno, ao perceber que não domina a transposição do conhecimento em questão, pode interromper a sequência de seu raciocínio nas aulas de cálculo, por exemplo, e comprometer seu êxito na compreensão de conceitos mais complexos que dão continuidade ao aprendizado.

As duas próximas questões são, em sua essência, muito parecidas. De fato, a equação polinomial envolvida é a mesma. As diferenças consistem no que diz respeito ao domínio da função e o enunciado da mesma. A primeira delas, trata de um problema de aplicação, relacionando o gráfico da função com a trajetória descrita pela bala.

As orientações de Brasil (2006) evidenciam a aplicação das funções com problemas do cotidiano, que relacionam grandezas, sugerindo que

O estudo de funções pode ser iniciado com uma exploração qualitativa das relações entre duas grandezas em diferentes situações: idade e altura; área do círculo e raio; tempo e distância percorrida; tempo e crescimento populacional; tempo e amplitude de movimento de um pêndulo, entre outras.

Questão 2. Uma bala é atirada de um canhão. A trajetória da bala descreve uma parábola de equação $y(x)=$ $-3 x^{2}+60 x$ (onde $x$ e $y$ são medidos em metros). Calcule o alcance do disparo da bala.

Dos 55 alunos que participaram da atividade respondendo o questionário, 33 alunos não resolveram esta questão. Isto significa que $60 \%$ não esboçou a solução. Destes, apenas 7 escreveram comentários do tipo "não lembro"ou "não sei". Apenas 11 (20\%) acertaram, e os demais, simplesmente escreveram a função na forma fatorada ou ainda erraram o cálculo algébrico. Novamente percebe-se que os alunos não conseguiram relacionar a interpretação prática da questão com o cálculo da raiz.

A próxima questão, apresenta a mesma função diretamente, sem contextualizá-la, solicitando apenas que sejam determinadas as raízes e o esboço do gráfico, isto é, sem que seja atribuído um sentido físico ao cálculo envolvido.

O objetivo das duas questões semelhantes é verificar se o aluno não apenas conhece o conceito abstrato de raiz e reconhece a forma geral do gráfico de uma função polinomial de grau dois, mas também identifica esse conceito na prática. Em um segundo momento, verificar se o aluno conseguiria identificar a semelhança das duas questões podendo até mesmo registrar este fato ao longo da solução.

Questão 3. Determine as raízes da função $f(t)=60 t-3 t^{2}$. Esboce o gráfico da função $f(t)$.

Foram 21 alunos que deixaram esta questão em branco. Alguns escreveram comentários como "não lembro"ou ainda "não sei"e um deles escreveu ainda "tenho dúvidas". O número de alunos que não respondeu a questão é cerca de 30\% menor do que a questão anterior. O fato da questão possuir um enunciado mais longo, onde a pergunta não explicita o cálculo que deve ser realizado desencoraja o aluno que sequer tenta interpretá-la. Isto contradiz o discurso presente na sala de aula, onde o estudante argumenta que não estuda, pois o conteúdo não serve para nada.

Apenas dois estudantes escreveram "sei fazer"sem esboçar nem parte da solução. De fato, cerca de $40 \%$ dos 
alunos que responderam o questionário e não esboçaram alguma tentativa de determinar as raízes e também o gráfico.

Dos 8 alunos que resolveram corretamente, 5 deles encontraram as raízes mas não esboçaram o gráfico. Um ponto positivo identificado foi o de que os alunos escreveram $f(t)=0$ mostrando que sabem qual é a equação que deve ser resolvida para obter as raízes e para isso utilizam a fórmula de Bhaskara.

Apenas 1 aluno identificou a semelhança com a questão 7, escreveu "são iguais"mas não resolveu.

Todos os demais que esboçaram uma tentativa de solução não conseguiram calcular corretamente as raízes. Dois deles esboçaram um gráfico que não se assemelha a uma parábola. Muitos ainda esboçaram a parábola côncava para cima, o que possivelmente tenha ocorrido a partir da simplificação incorreta $-t^{2}=(-t)^{2}$.

Percebeu-se que para os alunos que participaram da pesquisa, foi mais fácil calcular as raízes algebricamente do que identificá-las geometricamente como solicitado na questão 1 . Isto é, compreendem o cálculo de $f(t)$ para um $t$ dado, mas possuem dificuldades em identificar geometricamente o cálculo efetuado.

Após a aplicação do teste, percebe-se a necessidade de propor atividades que trabalhem os conceitos envolvidos para auxiliar na transição dos estudos de gráficos do Ensino Médio para o Ensino Superior no sentido de generalizar o estudo que é feito com funções afim e quadrática para funções polinomiais de grau maior que 2. Além disso, pretende-se contribuir para a transposição da visualização gráfica para a linguagem matemática, uma vez que a conversão de um registro numérico para um registro na forma algébrica ou gráfica (e vice-versa) é de grande importância para a compreensão de conceitos matemáticos.

\section{A importância das diferentes formas de representação de objetos matemáticos}

Considera-se fundamental que o estudante explore a conversão entre as diferentes representações dos objetos matemáticos a fim de facilitar a construção de conceitos e tornar a aprendizagem significativa. A transição entre as diferentes formas de registro pode ser uma dificuldade ao domínio completo dos conteúdos e a sua aplicação não apenas nas disciplinas de matemática.

Segundo Duval (2003),

Há uma pluralidade de registros de representação de um mesmo objeto, e a articulação desses diferentes registros é a condição para a compreensão em matemática, embora várias abordagens didáticas não levem em conta esse fato.

Duval afirma que é essencial dominar as diferentes representações de um objeto matemático, pois isso permite que o estudante mobilize entre formas distintas de representação e registro escolhendo aquela que se mostra mais adequada para determinada situação. Para que essa mobilidade ocorra, porém, é necessário o pleno domínio dos conceitos envolvidos. Esta questão torna-se paradoxal, pois percebe-se que os estudantes em sua maioria não possuem a apreensão conceitual dos objetos matemáticos considerados.

Uma pesquisa inicial identificou alguns obstáculos pedagógicos remanescentes do Ensino Básico. A partir desta identificação propõem-se atividades que envolvem os mesmos conceitos matemáticos em representações diferentes. As atividades propostas na seção 6 são uma estratégia pedagógica para o desenvolvimento das competências associadas ao conteúdo de funções polinomiais.

Um aspecto que este trabalho considera e que vai ao encontro da Teoria de Duval, é o de que o autor considera a conversão (entre as representações) uma atividade cognitiva fundamental. É importante destacar que apenas uma representação não garante que ocorra a aprendizagem. Segundo Oliveira e Pires (2012), para Duval, aprender matemática está condicionado ao ato de representar, tratar e converter registros de representação em um determinado nível cognitivo.

Por exemplo, dada uma função representada simbolicamente, deseja-se que o aluno, com o auxílio do software, consiga convertê-la para a representação gráfica. Este fato aparece, por exemplo, na Questão 1, onde deseja-se verificar como ocorre a conversão da representação cartesiana para a algébrica. As atividades propostas recorrem à diversidade de representações do mesmo objeto matemático, com o uso de diferentes registros de representação, não só para tratamentos do mesmo objeto, mas principalmente na conversão de registros nas diferentes formas de linguagem (aritmética, algébrica, entre outras). Acredita-se que tais transformações nos registros de representação podem auxiliar na caracterização do objeto matemático e desempenhar um papel importante na compreensão dos alunos.

Seguindo estas ideias e devido às dificuldades dos alunos, são propostas na seção 6 atividades com o software GeoGebra a fim de facilitar a aprendizagem dos conceitos acerca das funções polinomiais. 


\section{Atividades propostas}

Os softwares educacionais, de uma maneira geral, oferecem ao aluno um ambiente de trabalho no qual ele pode visualizar, conjecturar, investigar os resultados, validar suas conclusões a partir de suas próprias investigações e descobertas. Segundo Borba e Villarreal (2006), "a tecnologia computacional enfatiza a componente visual da matemática, mudando o status da visualização na educação matemática".

Ainda, D'Ambrosio (1986) afirma que

Com a intuição, a abstração e a formalização, a visualização é um dos vários processos colocados em interação para matematizar o mundo, buscando compreender a realidade, representando-a por meio de modelos. Ao mesmo tempo em que observa a realidade, o indivíduo, baseando-se nela, desenvolve novas ideias e novos objetos concretos, atuando nessa realidade e transformando-a.

Nesse sentido, as atividades propostas foram desenvolvidas utilizando o software GeoGebra. Segundo Tall (2004), à medida que os alunos operam com o software para manipular as funções representadas de forma algébrica e geométrica, essas representações propiciam a corporificação dos símbolos. O software permite, assim, estabelecer uma comunicação entre o mundo concreto e o mundo simbólico. No mundo simbólico, os conceitos, no caso, as funções, são representados por símbolos manipuláveis, e o símbolos passam a integrar os processos de manipulação e os próprios conceitos.

Para as atividades propostas, sugere-se que a turma use o aplicativo do GeoGebra para celular ou tablet disponível em https://www.geogebra.org. Para isso, supõe-se que o professor e a turma possuam conhecimentos básicos sobre os comandos do GeoGebra. As atividades podem ser realizadas em um laboratório de informática, para isso basta que o software esteja instalado nos computadores. Sugere-se que no início da aula o professor entregue uma folha impressa com as questões propostas, nas quais eles poderão anotar suas impressões e respostas, se necessário. O período estimado para aplicar as 4 atividades é de 2 horas aula, podendo ainda, cada atividade ser desenvolvida separadamente.

O professor poderá ler as atividades em voz alta. Acredita-se que o ideal é que os alunos realizem as tarefas individualmente e, em um segundo momento, incentiva-se a discussão em grupo. Todas as atividades podem ser aplicadas a turmas de Ensino Médio, no entanto, sugere-se que sejam aplicadas nas aulas iniciais de um curso introdutório de Cálculo Diferencial.

O professor deve interferir o mínimo possível durante a realização das atividades, procurando manifestar-se apenas em situações nas quais as conclusões obtidas pelos alunos estejam equivocadas, acrescentando perguntas que direcionem o raciocínio correto, sem fornecer respostas prontas.

É importante destacar que a atividade individual permite que o aluno visualize e reconheça as principais características de cada função estudada. O segundo momento, contribui para desenvolver a comunicação em linguagem matemática e permite compartilhar saberes, esclarecer algumas dúvidas e formalizar conceitos. Acreditase que tais etapas: a leitura, o uso do GeoGebra, a reflexão individual e o aprendizado compartilhado contribuem para a construção dos conhecimentos matemáticos desejados, permitindo, assim, que o estudante passe a operar os conceitos estudados simbolicamente e ingressem no mundo matemático formal.

Ao final da aula, sugere-se que os alunos entreguem as atividades para que o professor possa refletir sobre os resultados e repensar o plano de ensino para as aulas seguintes. Segundo Frota et al. (2013)

Vale destacar que uma ênfase no pensamento visual para aprender a comunicar ideias em cálculo depende de um novo papel do professor de matemática como aquele que: reflete sobre sua prática e desenvolve esforços para alterar o foco das tarefas que propõe; cria ambientes de aprendizagem que possibilitam a troca de experiências e a construção ou reconstrução de ideias matemáticas, utilizando tecnologias que podem ser consumidas e incorporadas, aos poucos, como ferramentas cognitivas; está consciente de que a utilização de determinada tecnologia em sala de aula depende do seu esforço pessoal para conhecer as potencialidades e as limitações do recurso tecnológico adotado, ou seja, depende de seu esforço pessoal em consumir e incorporar tecnologias, para empregá-las de maneira que mudem as formas de pensar e fazer matemática de seus alunos.

Utilizando o software GeoGebra, explora-se novamente a Questão 1, adaptando-a para o uso do aplicativo. O objetivo é que o aluno consiga relacionar a solução de uma equação ou inequação com o gráfico através de perguntas que o auxiliem a obter a resposta correta. 
Atividade 1 (Estudando uma Função Polinomial de grau 3). Responda as questões propostas com o auxílio do software GeoGebra.

1. No campo Entrada do GeoGebra, digite $f(x)=x^{3}-4 x^{2}+x+6$;

2. Escreva com suas palavras o que aparece na Janela de Visualização.

O gráfico é contínuo?

Intercepta o eixo $x$ ?

Intercepta o eixo $y$ ?

Quais as coordenadas dos pontos de intersecção?

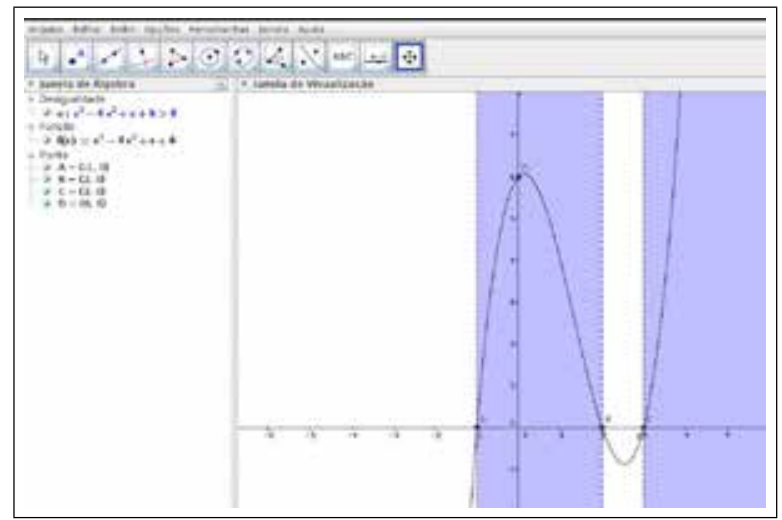

Figura 2: Solução para $f(x)>0$

3. Determine as raízes de $f(x)$.

Observe que as raízes são as abscissas dos pontos onde a função intercepta ou toca o eixo $x$.

Para confirmar sua resposta, digite Raiz[f] no campo Entrada.

Na janela de Álgebra aparecerão as coordenadas dos pontos onde o gráfico intercepta o eixo $x$.

Verifique que a ordenada desses pontos é zero.

4. Determine as coordenadas do ponto onde o gráfico intercepta o eixo $y$.

Observe que a abscissa deste ponto é zero.

Digite $(0, f(0))$ no campo Entrada e verifique se sua resposta está correta.

5. Para quais valores de $x$ a função $f(x)$ é positiva?

Note que $f(x)$ tem que assumir valores positivos, isto é, $f(x)>0$.

Para confirmar sua resposta, digite $f(x)>0$ no campo Entrada.

Note que os pontos do eixo $x$ interceptados pela região colorida representam o intervalo onde $f(x)>0$, isto é, $f(x)$ assume valores positivos nesse intervalo.

6. Antes de responder a próxima pergunta, oculte na janela de Álgebra a desigualdade $f(x)>0$.

7. Para quais valores de $x$ a função $f(x)$ é negativa?

Note que $f(x)$ tem que assumir valores negativos, isto é, $f(x)<0$.

Para confirmar sua resposta, digite $f(x)<0$ no campo Entrada.

Note que os pontos do eixo $x$ interceptados pela região colorida representam o intervalo onde $f(x)<0$, isto é, $f(x)$ assume valores negativos nesse intervalo.

As Figuras 2 e 3 ilustram as janelas de visualização do software GeoGebra para os itens 5 e 7. 
Com o auxílio do software GeoGebra, explora-se agora as Questões 2 e 3 apresentadas no teste de sondagem. O objetivo é que o aluno, a partir da representação gráfica, seja capaz de construir a solução da situação problema proposta a partir de investigações que o guiarão nessa construção.

Atividade 2 (Estudando a trajetória da bala do canhão). Uma bala é atirada de um canhão. A trajetória da bala descreve uma função cuja lei é $y(x)=-3 x^{2}+60 x$ (onde $x$ e $y$ são medidos em metros). Calcule o alcance do disparo da bala.

1. No campo Entrada do GeoGebra, digite $y=-3 x^{2}+60 x$;

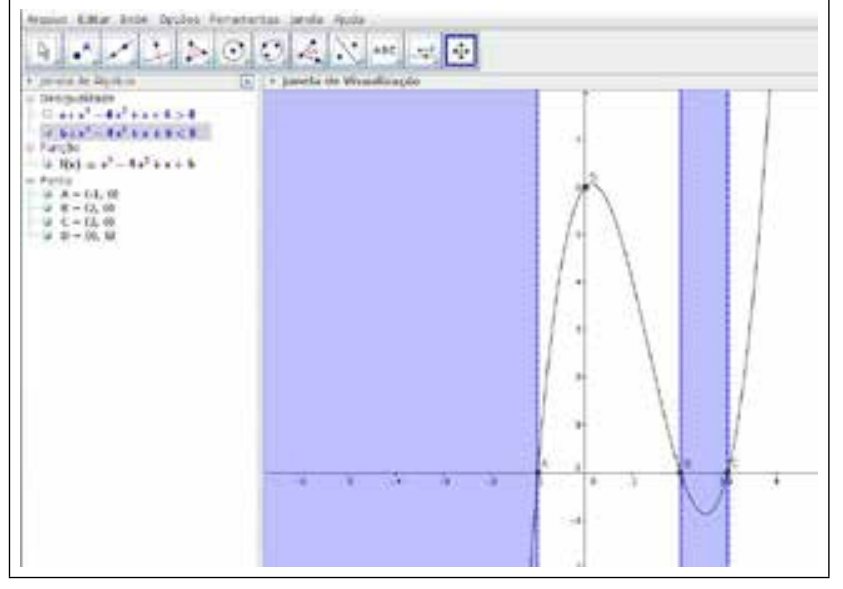

Figura 3: Solução para $f(x)<0$

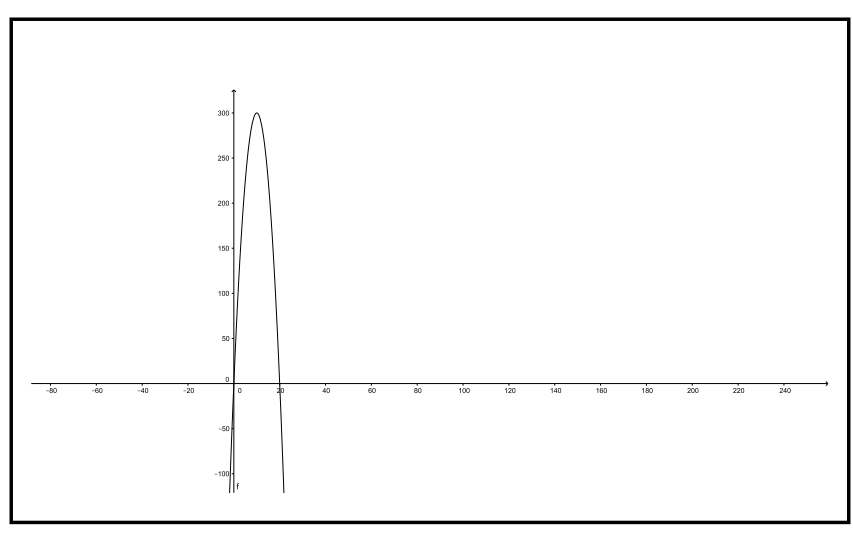

Figura 4: Gráfico de $y=-3 x^{2}+60 x$

2. Descreva com suas palavras como é a trajetória da bala de acordo com o que está representado na Janela de Visualização. Movimente a Janela de Visualização para obter mais detalhes.

A bala descreve uma trajetória reta? Uma trajetória circular? Ou seria uma trajetória parabólica?

A bala atinge uma altura máxima ou ela nunca pára de subir? Qual variável representa esta altura, $x$ ou $y$ ?

De acordo com o gráfico, de qual valor sobre o eixo $x$ parte a bala? Isto está correto dentro do contexto do problema?

A Figura 4 ilustra o gráfico de $y=-3 x^{2}+60 x$.

3. Agora, no campo Entrada, digite $y=$ Função[ $\left.-3 x^{2}+60 x, 0,20\right]$;

Houve alguma alteração na trajetória da bala? Quais?

O que representam os valores 0 e 20, sobre o eixo $x$ ?

Estes valores têm alguma relação com as raízes da função quadrática $y=-3 x^{2}+60 x$ ?

Como você descreveria o deslocamento da bala ao longo do eixo $x$, ou seja, para frente?

De quanto foi este deslocamento?

Portanto, podemos afirmar que o alcance do disparo da bala foi de quantos metros?

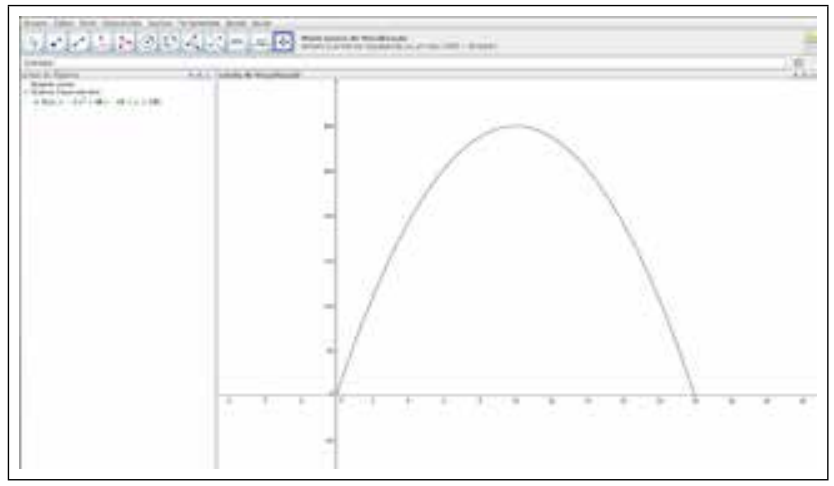

Figura 5: Gráfico de $y=-3 x^{2}+60 x, x \in[0,20]$

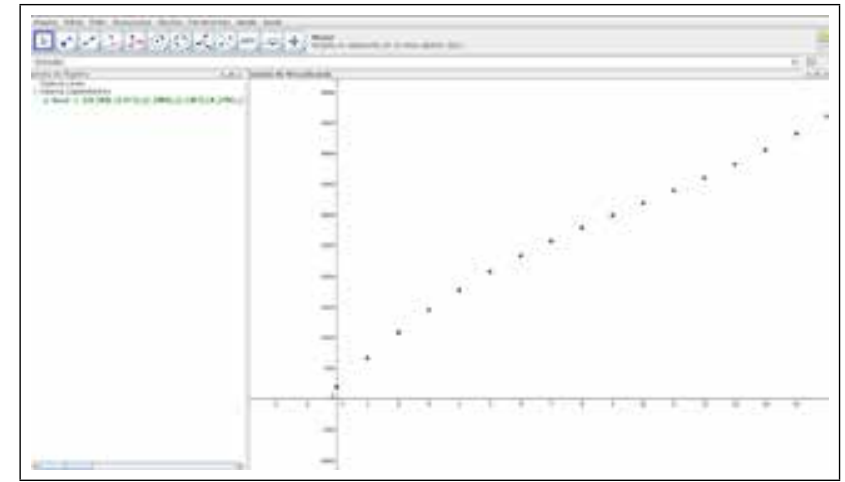

Figura 6: Gráfico de $C(q)$ 
A Figura 5 apresenta o gráfico de $y=-3 x^{2}+60 x$ restrita ao intervalo $[0,20]$.

Com o objetivo de uma melhor compreensão dos problemas que foram aplicados no teste de sondagem, a seguir, são propostas mais duas atividades, acerca do conteúdo de funções polinomiais de grau 3. Os objetivos são estudar o conceito de domínio discreto e explorar a representação algébrica e gráfica da função, respectivamente.

Atividade 3 (Explorando domínio discreto). Considere uma empresa em que o custo total de produção de canetas, em unidades monetárias (u.m.), é definido pela função $C(q)=q^{3}-30 q^{2}+500 q+200$.

1. Qual é o domínio da função $C(q)$ ? Qual é a imagem da função $C(q)$ ?

2. A empresa pode produzir um número negativo de canetas? A empresa pode produzir um número não inteiro de canetas?

3. No software GeoGebra, clique com o botão direito do mouse na Janela de Visualização, na opção Eixo X:Eixo $Y$, altere a escala do gráfico para $1: 500$.

4. Construa o gráfico da função $C(q)=q^{3}-30 q^{2}+500 q+200$.

5. Este gráfico representa a função custo total de produção da empresa?

6. Observe que no gráfico de $C(q)$ existem valores negativos tanto para o domínio quanto para a imagem. Dentro do contexto do problema, faz sentido obter $C(q)<0$ ? Com a representação feita no GeoGebra, a empresa pode produzir, por exemplo, 2,5 canetas?

7. Explorando o domínio Discreto no GeoGebra. No campo Entrada, digite Sequência $\left[\left(q, q^{3}-30 q^{2}+500 q+\right.\right.$ 200), $q, 0,20,1]$.

Observe que o custo deve ser considerado para valores inteiros de $q$, ou seja, o domínio de $C(q)$ é $\mathbb{Z}$. A imagem de $C(q)$ corresponde a um subconjunto de $[0,+\infty[$.

8. Determine o custo de produção de 10 canetas.

9. Calcule o custo total de produção da décima caneta.

A Figura 6 apresenta o gráfico de $C(q)$, com seu domínio discreto.

Atividade 4 (Investigando o gráfico de uma função polinomial de grau 3 na forma fatorada). Seja $f(x)=(x-$ 1) $(4 x-1)(4 x-3)$. Esboce o gráfico de $f(x)$ no GeoGebra, digitando no campo de entrada a função $f(x)$ e responda as questões.

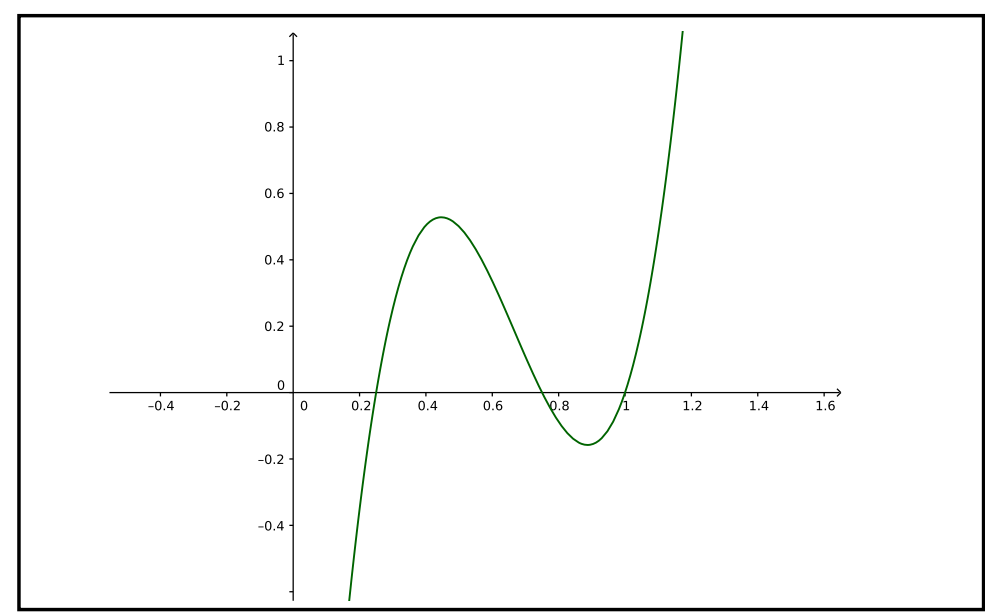

Figura 7: Gráfico de $f(x)=(x-1)(4 x-1)(4 x-3)$

1. A função $f(x)$ troca de sinal?

2. Em quais pontos esta troca de sinal acontece?

3. O que representam os pontos identificados no item 2?

4. Determine, graficamente, a intersecção de $f(x)$ com o eixo $y$.

5. Como você calcularia o valor determinado no item 4 ?

Esta atividade foi adaptada de Giraldo et al. (2013). A Figura 7 mostra o gráfico da Atividade 4. 


\section{Discussão da aplicação da atividade 4}

Uma vez identificadas as dificuldades dos alunos no Ensino Superior ao trabalhar com as funções polinomiais, a atividade 4 foi aplicada a fim de investigar a metodologia proposta e avaliar a capacidade do aluno em ler e interpretar um gráfico de uma função polinomial de grau três e extrair dele as informações solicitadas.

A atividade foi realizada no mês de junho do ano de 2015 no laboratório de informática da Universidade, com duração de 1 hora aula e contou com a presença de 16 alunos de uma disciplina introdutória ao Cálculo Diferencial. É importante ressaltar que estes discentes já haviam realizado o teste de sondagem descrito na seção 3 .

Os alunos receberam um material impresso contendo a atividade, digitaram a função polinomial no campo Entrada do software GeoGebra, analisaram o gráfico construído e formularam, individualmente, suas conclusões. A atividade desenvolvida foi preparada seguindo um caráter sistemático, ou seja, as respostas a cada item da atividade são gradativas, permitindo ao aluno observar, analisar e registrar suas observações, a fim de construir e fixar os conceitos sobre o conteúdo. Ao final da atividade, os discentes entregaram ao professor os resultados obtidos a partir de suas observações.

É importante destacar que, neste momento, os alunos já haviam estudado os tópicos principais relacionados a funções polinomiais, tais como: identificação de um polinômio; grau e valor numérico; igualdade de polinômios; operações com polinômios: adição, subtração, multiplicação e divisão; Teorema do Resto; Teorema de D'Alembert; dispositivo prático de Briot-Ruffini; equações polinomiais; Teorema Fundamental da Álgebra; Teorema da Decomposição em Frações Parciais; multiplicidade de uma raiz; Relações de Girard e representação gráfica de um polinômio.

Na sequência, são apresentados os resultados obtidos para cada um dos itens solucionados pelos discentes.

No primeiro item, o aluno deveria visualizar no gráfico construído com o software GeoGebra que a imagem da função $f(x)$ assume ora valores positivos, ora negativos. E assim, concluir que $f(x)$ troca o seu sinal. Com relação a este item, todos os 16 acadêmicos responderam corretamente a questão.

No item 2, o aluno é questionado sobre os pontos onde ocorre a troca de sinal da função $f(x)$. Para esta pergunta, apenas 1 aluno determinou de forma incorreta os pontos. Os demais acertaram a questão. É importante mencionar que 3 alunos identificaram, ainda, o sinal da função e o intervalo correspondente.

No item 3, o aluno deveria relembrar o conceito de raiz de uma função e identificá-las no gráfico. Para este item, 15 acadêmicos responderem corretamente e apenas 1 aluno não relacionou os pontos com as raízes da função.

Nos itens 4 e 5, o aluno deveria a partir da construção gráfica, identificar o ponto de intersecção com o eixo das ordenadas e determinar tal ponto analiticamente considerando $x=0$ na função $f(x)$. No quarto item, 12 alunos determinaram corretamente a intersecção da função com o eixo y observando o gráfico construído. Quatro alunos não responderam a questão. Este foi o item com a menor taxa de acerto, $75 \%$. No item 5 , apenas 1 aluno deixou a questão em branco. Os demais calcularam o valor corretamente.

\section{Conclusões}

Segundo os Parâmetros Curriculares Nacionais do Ensino Médio (PCNEM), cujo texto encontra-se em Brasil (1998), o conceito de função desempenha um papel importante para descrever e estudar através da leitura, interpretação e construção de gráficos, o comportamento de fenômenos do cotidiano, como de outras áreas do conhecimento. Com o ensino de gráficos de funções polinomiais deseja-se garantir que o aluno tenha autonomia para interpretar um gráfico e reconhecê-lo em situações diversas pois a compreensão de um conceito matemático em geral está relacionado à capacidade do aluno em migrar de uma forma de representação matemática para a outra. É importante reconhecer as diferentes linguagens que a matemática apresenta e aprender à estabelecer relações entre as diferentes representações de um objeto matemático.

Segundo (Dierings, 2014), salvo as escolas técnicas, que focam seus ementários em determinadas áreas, a maioria das escolas brasileiras trabalha, ou pelo menos deveria trabalhar, na "preparação "de um aluno com condições mínimas de acompanhar um Ensino Superior, qualquer que seja a área. Acredita-se que o papel da Escola e também da Universidade é preparar os estudantes para enfrentar situações simples do cotidiano, como a leitura e interpretação de um gráfico em um jornal diário ou ainda formular um parecer próprio a partir das informações obtidas no mesmo.

Se a Escola Básica não possui recursos para práticas alternativas de ensino de gráficos, a Universidade de maneira geral possui uma infraestrutura adeauada e professores aualificados para proporcionar tais práticas. Os laboratórios 
de informática estão habilitados com softwares gratuitos e a internet disponibiliza tutoriais simples para iniciantes. Dessa forma, uma alternativa para apresentar ou ainda revisitar os conceitos é utilizar ferramentas computacionais no ensino das disciplinas iniciais dos cursos de Ciências Exatas e Engenharias. A formalização dos conceitos a partir da construção geométrica do gráfico leva a uma aprendizagem significativa, pois o aluno tem a possibilidade de repetir o processo, se necessário, aprendendo, assim, com seus próprios erros, até tornar a construção familiar.

No Ensino Básico, uma abordagem qualitativa e profunda não deve ser feita dentro da parte flexível do currículo, como opção específica de cada escola. O estudo de gráficos, de maneira geral, é fundamental para o sucesso acadêmico do aluno nos cursos de Ciências Exatas e Engenharias e um recurso fundamental para tornar o aluno um cidadão crítico capaz de analisar e argumentar em diferentes situações do cotidiano representadas por gráficos de funções polinomiais. A fim de dar continuidade e fundamentar quantitativamente a proposta de inclusão de atividades computacionais em disciplinas básicas de Matemática no Ensino Superior, no primeiro semestre de 2015 foram introduzidas aulas de laboratório para abordar conceitos acerca de funções polinomiais, exponenciais e logarítmicas. Futuramente, pretende-se disponibilizar os dados obtidos e comparar o desempenho destes alunos com o de anos anteriores.

\section{Referências}

Anton, H. (2000). Cálculo: um novo horizonte, vol 1, 60 edn. Bookman.

Ballejo, C. C. (2009). O uso de software no ensino de funções polinomiais no ensino médio. Dissertação de Mestrado, Universidade Federal do Estado do Rio Grande do Sul, Porto Alegre, trabalho de Conclusão de Curso.

Borba, M., Villarreal, M. (2006). Humans-with-media and the reorganization of mathematical thinking: information and communication technologies, modeling, experimentation and visualization, Springer, Cap. Visualization, mathematics education and computer environments.

Brasil (1998). Parâmetros Curriculares Nacionais: Matemática. Ministério da Educação e Cultura, Brasília, URL http: //portal.mec.gov.br/seb/arquivos/pdf/matematica.pdf.

Brasil (2006). Orientações Curriculares para o Ensino Médio. Ministério da Educação e Cultura, Brasília.

Brasil (2016). Instituto nacional de estudos e pesquisas educacionais anísio teixeira. URL http://enem.inep.gov .br/.

Catani, A. M., Oliveira, J. F., Dourado, L. F. (2001). Política educacional, mudanças no mundo do trabalho e reforma curricular dos cursos de graduação no brasil. Educação e Sociedade, 22(75), 67-83.

Cavasotto, M., Portanova, R. (2008). Reflexões sobre as dificuldades na aprendizagem de cálculo diferencial e integral. Em: Anais da III Mostra de Pesquisa da Pós-Graduação PUCRS, URL http://www.pucrs.br/edipucrs/ online/IIImostra/EducacaoemCienciaseMatematica/62352\%20-\%20MARCELO\%20CAVASOTTO. pdf.

Coxford, A. F., Shulte, A. P. (1994). As idéias da álgebra. Atual, São Paulo, traduzido por Hygino H. Domingues.

da Cruz Mendes, S. C., Fainguelernt, E. K., Rodrigues, C. K. (2012). Geogebra e a família dos números metálicos. Em: Anais I Conferência Latino Americana de GeoGebra, pp. 160-171.

Cury, H. (2007). Análise de erros: o que podemos aprender com as respostas dos alunos, $6^{\circ}$ edn. Autêntica, Porto Alegre.

D’Ambrosio, U. (1986). Da realidade à ação: reflexões sobre educação matemática. Sammus, Campinas.

Dante, L. R. (2010). Matemática: contexto e aplicações, vol 1. Atica, São Paulo.

Dierings, A. R. (2014). Ensino de polinômios no ensino médio - uma nova abordagem. Dissertação de Mestrado, Universidade Federal de Santa Maria, Santa Maria, dissertação.

Duval, R. (2003). Aprendizagem em Matemática: Registros de Representação Semiótica, Papirus, Cap. Registros de Representações Semióticas e Funcionamento Cognitivo da Compreensão em Matemática.

Edwards, L. H. (2006). Cálculo, $8^{0}$ edn. Mcgraw Hill, São Paulo.

Eisenberg, T., Dreyfus, T. (1994). On understanding how students learn to visualize function transformations. Research on Collegiate Mathematics Education, 1, 45-68. 
Fainguelernt, E. K., Nunes, K. R. A. (2012). Matemática: práticas pedagógicas para o ensino médio. Penso, Porto Alegre. Fernandes, V. S., Silva, J. D., Mabelini, O. D. (2005). Matemática para o ensino médio. IBEP, São Paulo.

Filho, B. B., Silva, C. X. (2000). Matemática aula por aula. FTD, São Paulo.

Fleming, D. (2006). Cálculo A: funções, limites, derivação e integração, $6^{\circ}$ edn. Pearson Prentice Hall.

Frota, M. C., Carvalho, A. M., Bianchini, B. (2013). Marcas da educação matemática no ensino superior. Papirus, São Paulo.

Giovanni, J. R., Bonjorno, J. R., Junior., J. R. G. (2005). Matemática Completa. Editora FTD, São Paulo.

Giraldo, V., Caetano, P. A. S., Mattos, F. P. (2013). Recursos Computacionais no Ensino de Matemática, $1^{\circ}$ edn. SBM.

Guidorizzi, H. (2006). Um curso de Cálculo, $5^{\circ}$ edn. LTC.

Hoffmann, L. D., Bradley, G. L. (1999). Cálculo: um curso moderno e suas aplicações, $6^{\circ}$ edn. LTC, Rio de Janeiro.

Lima, E. L. (1998). Coordenadas no Espaço, $3^{\circ}$ edn. SBM, Rio de Janeiro.

Lima, E. L., Wagner, E., Carvalho, P. C. P., Morgado, A. C. (2001). A matemática do ensino médio, vol 3, $6^{\circ}$ edn. SBM, Rio de Janeiro.

Malta, I. (2004). Linguagem, leitura e matemática. EDIPUCRS, Porto Alegre.

Munem, M. A., Foulis, D. J. (1982). Cálculo, vol 1. LTC, Rio de Janeiro.

Nasser, L., Sousa, G., Torraca, M. (2012). Transição do ensino médio para o superior: como minimizar as dificuldades em cálculo? Em: Atas do V Seminário Internacional de Pesquisa em Educação Matemática (em CD), Petrópolis, RJ.

Oliveira, P. C., Pires, R. F. (2012). O conceito de função na educação básica vis registros de representação semiótica. Revista Reflexão e Ação, 20(2), 215-239.

Pereira, A., Shitsuka, C., Júnior, C. A., Shitsuka, D., Paiva, E., Rosetti, H., Silveira, I., Schimiguel, J., Calejon, L., Lugli, L., Shitsuka, R. (2012). Estudos e Aplicações em Ensino e Aprendizagem de Matemática. Ciência Moderna.

Rezende, W. M. (2003). O ensino de cálculo: dificuldades de natureza epistemológica. Dissertação de Mestrado, Universidade Federal do Estado de São Paulo, São Paulo, tese (Doutorado em Educação).

Rodriguez, B., Meneghetti, C. M. S., Poffal, C. A. (2015). Estudo do perfil dos alunos do curso de matemática aplicada - bacharelado: entendendo as razões para o baixo rendimento dos acadêmicos. Ciência e Natura, 37(1), $151-162$.

Simmons, G. (1987). Cálculo com Geometria Analítica, vol 1. Mc Graw-Hill, São Paulo.

Soares, E. M., Sauer, L. Z. (2004). Disciplinas Matemáticas em Cursos Superiores: reflexões, relatos, propostas, EDIPUCRS, Cap. Um novo olhar sobre a aprendizagem de matemática para a engenharia.

Tall, D. (2004). Intoduction to the three worlds of mathematics. Em: Proceedings of the 28th Conference of the International Group for Psychology of Mathematics Education, Bergen, Noruega.

Thomas, G. B. (2009). Cálculo, $11^{\circ}$ edn. Pearson - Addison Wesley, colaborador: Maurice Weir, Joel Hass, Frank R. Giordano; Tradução: Luciana do Amaral Teixeira, Leila Maria Vasconcellos Figueiredo. 